\title{
Hospitalización, multimorbilidad y edad avanzada: ¿realmente actuamos bien?
}

\author{
Hospital admissions, multimorbidity and elderly patients: Are we really going in the right direction?
}

Juan Carlos Piñeiro-Fernández, Emilio Casariego-Vales

Servicio de Medicina Interna. Hospital Universitario Lucus Augusti. SERGAS. España

En España, el envejecimiento poblacional es un hecho (el censo de julio de 2020 incluye 569.277 personas de 90 o más años), los avances sanitarios no cesan y la expectativa de vida aumenta cada año'. Lo que sin duda es un éxito a nivel poblacional, tiene un gran impacto en la organización de la atención sanitaria y nos obliga a realizar muchos cambios, y muy complejos. Este fenómeno tiene una significativa repercusión en el ámbito hospitalario, ya que la mitad de todas las estancias son de población anciana o muy anciana ${ }^{2}$ y se han incrementado exponencialmente los ingresos repetidos de pacientes con multimorbilidad ${ }^{3}$. Los ingresos de pacientes nonagenarios ${ }^{4,5}$ o centenarios ${ }^{6}$ hace tiempo que no representan un fenómeno aislado, sino más bien una norma que ha llegado para quedarse. Además, la pandemia por la COVID-19 ha puesto de nuevo de manifiesto el riesgo aumentado de hospitalización y muerte en este grupo etario, relacionadas con la limitación funcional y fragilidad propia de la senescencia, que se agravan considerablemente durante el ingreso?.

En este mismo número de Galicia Clínica ${ }^{5}$, García Ibarbia et al analizan retrospectivamente las características clínicas y la evolución hospitalaria de 932 pacientes con 90 o más años ingresados en un servicio de Medicina Interna durante el año 2017. Como hallazgos más relevantes destacan: el número de pacientes nonagenarios (representan el $22,5 \%$ de los ingresos anuales en su servicio), la importancia de la multimorbilidad (en el $78,9 \%$ de los pacientes coexistieron 4 o más enfermedades), el notable grado de dependencia (Índice de Barthel medio: dependencia moderada) y la elevada tasa de mortalidad (21.7\% durante el ingreso y 55,3\% al año). Se constata que el perfil de los pacientes de mayor edad ingresados en nuestros hospitales es el de personas con elevadas necesidades asistenciales, a lo hay que añadir otra característica muy relevante como es su alta tasa de reingresos ${ }^{3}$.

A pesar de que llevamos más de dos décadas "preparándonos" para este fenómeno, la atención hospitalaria dirigida a estos pacientes, que precisan tantos recursos sanitarios, sigue siendo un reto sin resolver ${ }^{\text {. }}$ Conocemos el problema, su importancia, sus características y sus repercusiones, pero el cómo abordarlo sigue sin tener una respuesta clara y concreta por parte de la administración sanitaria 8 . Sin embargo, existen medidas conocidas y bien definidas que pueden aportar mejoras sustanciales ${ }^{7,9}$.

El primer aspecto es reconocer que ningún paciente adquiere la multimorbilidad de forma sobrevenida, repentina y sin margen de maniobra. De hecho, con una red asistencial tan universal y estructurada como la del SERGAS, no debiera ser demasiado complicado establecer las necesidades asistenciales adecuadas en las fases precoces de la evolución de sus enfermedades crónicas. De este modo, podremos planificar la atención teniendo en cuenta las características de cada paciente, incluso antes de su primer ingreso hospitalario. Esto nos permite gestionar planes sistemáticos acordes a la complejidad de cada caso y que tengan en cuenta las necesidades personales y sociales, dedicados ante todo a mejorar la calidad de vida, la situación funcional, controlar la multimorbilidad y reducir los reingresos, con una atención predominantemente ambulatoria y la creación de equipos de trabajo (médicos y Enfermería gestora de casos) estables, intentando simplificar la asistencia ${ }^{3,4,7,8}$. Para ello, es imprescindible establecer una colaboración real, eficaz y continuada entre la Atención Primaria, la Atención Hospitalaria y los Servicios Sociales ${ }^{9,10}$. Nadie duda que la Atención Primaria es el eje pivotal en el seguimiento ambulatorio de estos pacientes, pero hace falta una planificación realista, sencilla y sensata de la relación con el ámbito hospitalario, donde los internistas podemos ofrecer el soporte adecuado para los pacientes de mayor complejidad ${ }^{9}$. Parece claro que esto debe de ser así pero, hasta la fecha, ni la planificación ni los resultados han sido convincentes.

El segundo aspecto a considerar es: ¿debemos cambiar la actuación médica durante el ingreso hospitalario? La elevada presión asistencial, la falta de formación específica y la pobre coordinación conducen a una inercia que perpetúa el abordaje en exclusiva del problema causante del ingreso, dedicándole menos atención a los problemas fundamentales. Sin embargo, el ingreso es una oportunidad única para coordinar la asistencia, garantizando una visión holística e identificando la gravedad y las necesidades reales del paciente ${ }^{10}$. Diseñar y planificar estas acciones, teniendo en cuenta los deseos del paciente, no siempre es 


\section{Conocemos el problema, hay acuerdos sobre la necesi- dad de reformas, existen soluciones plausibles, pero el tiempo pasa y no se cumplen las promesas...}

fácil'11. Por una parte, son situaciones de elevada complejidad que hacen imprevisible su evolución ${ }^{3,7}$. Por otra, no disponemos de un soporte científico sólido para su seguimiento ya que son pacientes excluidos de los ensayos clínicos y no están bien representados en las guías de práctica clínica habituales ${ }^{11}$, de modo que es preciso diseñar protocolos de actuación durante el ingreso, tanto para este subgrupo de pacientes como para los clusters de enfermedades más habituales $^{12}$. Por último, no siempre es posible aumentar el tiempo de supervivencia dado que en muchos casos nos encontramos con enfermos que se encuentran en estadios muy avanzados de sus enfermedades crónicas, por lo que es necesario centrar el objetivo debiendo tomar decisiones personalizadas en función de las posibilidades médicas, prioridades y necesidades del enfermo, evitando el encarnizamiento terapéutico o el nihilismo ${ }^{6,11}$.

En tercer lugar, es imprescindible planificar de manera sistemática los cuidados al alta para garantizar la continuidad asistencial. En nuestra experiencia, la implantación de programas de atención personalizada y proactiva al alta en los pacientes de mayor complejidad, ofrece beneficios notables ${ }^{7}$. Para ello, es preciso disponer de equipos hospitalarios que integren la Enfermería como gestora de casos o la telemedicina como herramientas esenciales, contar con unidades funcionales de altas prestaciones como los hospitales de día o la Hospitalización a Domicilio, fortalecer la asistencia compartida con los equipos de Atención Primaria y coordinarse de forma eficaz con los Servicios Sociales ${ }^{8,9,10}$. Para llevar a cabo este objetivo, es fundamental establecer una organización diferente, con una actuación global, planes realistas y presupuestos adecuados que engloben a todas las administraciones. Sin esta coordinación y liderazgo nunca tendremos el éxito necesario.

Por consiguiente, en estos momentos, la Medicina Interna tiene una posición privilegiada para impulsar cambios profundos como los señalados para la asistencia de los pacientes muy complejos y/o muy ancianos. En paralelo, existe otra necesidad organizativa aún mayor. La Sociedad Española de Medicina Interna ha participado, con otras muchas entidades, en el desarrollo del denominado "Hospital del futuro"10. En sus conclusiones se insiste en la necesidad de crear redes que pongan (por fin) al paciente en el centro de la atención, en crear sistemas alternativos a la hospi- talización convencional que aseguren la continuidad asistencial y que prioricen los cuidados ambulatorios, terminando con la fragmentación de la asistencia ${ }^{8}$. El "Hospital del Futuro" debe considerarse como un componente más de la "Atención Sanitaria del Futuro"10. Pero mientras hablamos del "futuro", la realidad es que los pacientes que necesitan todos estos cambios ya están aquí y conviven con nosotros.

Por todo ello, los clínicos demandamos que las reformas organizativas se hagan con urgencia. Es evidente que la solución no es simple, pero sí es posible y en su desarrollo deben tomar parte activamente todos los implicados, siendo la única manera de alcanzar cambios organizativos, asistenciales y formativos profundos. Conocemos el problema, hay acuerdos sobre la necesidad de reformas, existen soluciones plausibles, pero el tiempo pasa y no se cumplen las promesas...¿a qué estamos esperando?

\section{BIBLIOGRAFÍA}

1. Instituto Nacional de Estadística - Población residente por fecha, sexo, grupo de edad y nacionalidad. [Consultado el: 12/06/2021]. Disponible en: https://www.ine. es/jaxiT3/Datos.htm?t=9689

2. Pérez Díaz J, Abellán García A, Aceituno Nieto P, Ramiro Fariñas D. Un perfil de las personas mayores en España, 2020. Indicadores estadísticos básicos. Madrid, Informes Envejecimiento en red n² 25, 39p. [Consultado el: 15/06/2021]. Disponible en: http://envejecimiento.csic.es/documentos/documentos/enredindicadoresbasicos2020.pdf.

3. Matesanz-Fernández M, Monte-Secades R, İñiguez-Vázquez I, Rubal-Bran D, Guerrero-Sande H, Casariego-Vales E. Characteristics and temporal pattern of the readmissions of patients with multiple hospital admissions in the medical departments of a general hospital. Eur J Intern Med. 2015;26(10):776-81.

4. Singer M, Conde-Martel A, Hemmersbach-Miller M, Ruiz-Hernández JJ, Arencibia Borrego J, Alonso Ortiz B. Mortality hospital of nonagenarian patients in Internal Medicine. Rev Clin Esp (Barc). 2018;218(2):61-65.

5. Romay-Lema E, Piñeiro-Fernández JC, Ventura-Valcárcel P, Iñiguez-Vázquez I, Gómez-Méndez R, Rabuñal-Rey R, et al. Hospitalización en pacientes centenarios en Galicia en el periodo 2004-2013. Galicia Clin. 2017; 78 (1): 7-10

6. García Ibarbia C, Hernández Hernández JL, Olmos Martínez JM, Narcis Nan Nan D Características Epidemiológicas y Clínicas de los Pacientes Nonagenarios Hospitalizados en un Servicio de Medicina Interna de un Hospital Terciario. Galicia Clin 2021; 82-3: Galicia Clin 2021; 82-3: 142-145

7. Berntsen GKR, Dalbakk M, Hurley JS, Bergmo T, Solbakken B, Spansvoll L, et al. Person-centred, integrated and pro-active care for multi-morbid elderly with advanced care needs: a propensity score-matched controlled trial. BMC Health Serv Res. 2019;19(1):682.

8. Wagner EH. Organizing Care for Patients With Chronic IIIness Revisited. Milbank Q. 2019;97(3):659-664.

9. Casariego-Vales E, Zapatero-Gaviria A, Elola-Somoza FJ, en nombre del Comité de Estándares de Unidades de Medicina Interna. La Medicina Interna del siglo XXI: estándares de organización y funcionamiento. Rev Clin Esp. 2017;217:526---33

10. Gómez Huelgas R, Díez Manglano J, Carretero Gómez J, Barba R, Corbella X, García Alegría J, et al. The Hospital of the Future in 10 points. Rev Clin Esp (Barc). 2020;220(7):444-449.

11. Tinetti ME, Costello DM, Naik AD, Davenport C, Hernandez-Bigos K, Van Liew JR, et al. Outcome Goals and Health Care Preferences of Older Adults With Multiple Chronic Conditions. JAMA Netw Open. 2021;4(3):e211271.

12. Matesanz-Fernández M, Seoane-Pillado T, Iñiguez-Vázquez I, Suárez-Gil R, Pértega-Díaz S, Casariego-Vales E. Description of multimorbidity clusters of admitted patients in medical departments of a general hospital. Postgrad Med J. 2021:postgradmedj-2020-139361. 